\title{
DECAY AND ASYMPTOTIC BEHAVIOR OF SOLUTIONS OF THE KELLER-SEGEL SYSTEM OF DEGENERATE AND NONDEGENERATE TYPE
}

\author{
TAKAYOSHI OGAWA \\ Mathematical Institute, Tohoku University \\ Sendai, 980-8578, Japan \\ E-mail: ogawa@math.tohoku.ac.jp
}

\begin{abstract}
We classify the global behavior of weak solutions of the Keller-Segel system of degenerate and nondegenerate type. For the stronger degeneracy, the weak solution exists globally in time and has a uniform time decay under some extra conditions. If the degeneracy is weaker, the solution exhibits a finite time blow up if the data is nonnegative. The situation is very similar to the semilinear case. Some additional discussion is also presented.
\end{abstract}

\section{Keller-Segel system}

1.1. Survey for nondegenerate case. This note concerns the temporal behavior of a global solution of the degenerate parabolic elliptic system. Before introducing the problem we consider, let us begin with the original model of the chemotaxis called the Keller-Segel system introduced in [14]. The semilinear type of the original Keller-Segel system is the following for $\lambda \geq 0$

$$
\left\{\begin{aligned}
\partial_{t} u-\Delta u+\nabla(u \nabla \psi)=0, \quad x \in \mathbb{R}^{n}, t>0, \\
\partial_{t} \psi-\Delta \psi+\lambda \psi=u, \quad x \in \mathbb{R}^{n}, t>0, \\
u(0, x)=u_{0}(x), \quad x \in \mathbb{R}^{n}, \\
\psi(0, x)=\psi_{0}(x), \quad x \in \mathbb{R}^{n} .
\end{aligned}\right.
$$

Here the unknown function $u(t, x): \mathbb{R}_{+} \times \mathbb{R}^{n} \rightarrow \mathbb{R}_{+}$denotes the density of a mucus amoeba and $\psi(t, x): \mathbb{R}_{+} \times \mathbb{R}^{n} \rightarrow \mathbb{R}$ stands for the potential of chemical substances. In order to exploit the contrast between the existence and nonexistence of solutions, JägerLuckhaus [12], Wolansky [38] and Nagai [20] considered the parabolic-elliptic version of the above system:

2000 Mathematics Subject Classification: Primary 35K65; Secondary 35K15, 35K55, 58J35.

The paper is in final form and no version of it will be published elsewhere. 


$$
\left\{\begin{array}{l}
\partial_{t} u-\Delta u+\nabla(u \nabla \psi)=0, \quad x \in \mathbb{R}^{n}, t>0, \\
-\Delta \psi+\lambda \psi=u, \quad x \in \mathbb{R}^{n}, t>0 \\
u(0, x)=u_{0}(x), \quad x \in \mathbb{R}^{n} .
\end{array}\right.
$$

The asymptotic behavior of solutions for this system has been studied in detail in [12], [40], [1], [21], [9]. In fact, the system (1.2) has a strong connection with the self-interacting particles studied largely by Biler ([1], [2] and reference therein).

The above systems are also connected to a simplest model equation of the semiconductor device of bipolar type (cf. [19], [13]):

$$
\left\{\begin{array}{l}
\partial_{t} n-\Delta n-\nabla(n \nabla \psi)=0, \quad x \in \mathbb{R}^{n}, t>0, \\
\partial_{t} p-\Delta p+\nabla(p \nabla \psi)=0, \quad x \in \mathbb{R}^{n}, t>0, \\
-\Delta \psi=\varepsilon(p-n)+g, \quad x \in \mathbb{R}^{n}, t>0, \\
n(0, x)=n_{0}(x), \quad p(0, x)=p_{0}(x), \quad x \in \mathbb{R}^{n},
\end{array}\right.
$$

where $n(t, x)$ and $p(t, x)$ denote the density of the negative and positive charges, respectively, and $g(x)$ denotes the background charge density which is a given function. When the background charge can be neglected, the equation is considered as the two species version of the Keller-Segel model except the sign of the nonlinear interaction. The semiconductor device model chooses a stabler sign of the nonlinearity that makes the system admit large data global solutions. Note that the unstable case, there is an analogous blow up result for the above two species system (see Kurokiba-Ogawa [17] and Kurokiba-Nagai-Ogawa [16]). In both cases (1.2) and (1.3), the critical case for the equation is $n=2$ from the scaling point of view. This corresponds to the well known Fujita exponent $1+2 / n$ for the semilinear heat equation $([10])$, and in the two dimensional case the quadratic nonlinearity exactly corresponds to the critical situation. The existence, uniqueness and regularity theory for the corresponding problem in a bounded domain have already been done by many authors. Here we concentrate on the Cauchy problem in $\mathbb{R}^{2}$ to examine the scaling invariance point of view.

The results for the global existence for the Keller-Segel system (1.1) can be summarized as follows:

TheOrem $1.1([23])$. Let $\lambda>0$ be constant and $n=2$. Suppose $\left(u_{0}, v_{0}\right) \in\left(L^{1}\left(\mathbb{R}^{2}\right) \cap\right.$ $\left.L^{2}\left(\mathbb{R}^{2}\right)\right) \times H^{1}\left(\mathbb{R}^{2}\right)$ are positive. Then under the condition either for $(1.1)$,

$$
\int_{\mathbb{R}^{2}} u_{0}(x) d x<4 \pi
$$

or for $(1.2)$

$$
\int_{\mathbb{R}^{2}} u_{0}(x) d x<8 \pi
$$

the positive solution to (1.1) (or (1.2)) exists globally in time. Namely $(u, v) \in C([0, \infty)$; $\left.\left(L^{2} \cap L^{1}\right) \times\left(H^{1} \cap L^{1}\right)\right) \cap C^{1}\left((0, \infty) ; H^{2} \times H^{2}\right)$ and it satisfies that for all $T>0$, there exists a finite constant $C=C(T)$ such that

$$
\int_{\mathbb{R}^{2}}\{(1+u(t)) \log (1+u(t))\}+\frac{1}{2}\|\nabla v(t)\|_{2}^{2}+\frac{1}{2} \lambda\|v(t)\|_{2}^{2} \leq C(T), \quad t \in[0, T] .
$$


In both cases, the role of the generalized free energy (1.6) is important to obtain the time a priori estimate for the solutions. Note that it has been already proved that if the initial data satisfies

$$
\int_{\mathbb{R}^{2}} u_{0}(x) d x>8 \pi
$$

then the positive solution blows up in a finite time (cf. Biler [1], Nagai [20] and NagaiSenba-Yoshida [25]).

On the other hand, to discuss the analogous result for the simpler system $\lambda=0$ of (1.2) we encounter a different kind of technical difficulty. For this case, it is also known that the solution with $u_{0} \geq 0$ blows up in a finite time if $\int_{\mathbb{R}^{2}} u_{0}(x) d x>8 \pi$ (Biler [1] Nagai [20], [22] and Nagai-Senba-Yoshida [24]). For the whole space case, the restriction that the solution has the finite second moment $\int_{\mathbb{R}^{2}}|x|^{2} u(t) d x<\infty$ is removed by the scaling method in Kurokiba-Ogawa [17].

When the domain is bounded in $\mathbb{R}^{2}$ with the Neumann boundary condition, SenbaSuzuki [30] showed that the $L^{1}$ density exhibits a concentration with the measure $8 \pi \delta_{0}$ if the data is radially symmetric. This can be generalized to the nonradial case by SenbaSuzuki [31].

The second system (1.2) with $\lambda=0$ also has an analogous property. However, the proof of the global existence is rather complicated since the behavior of the solution of the second equation is different from the first one. Namely we cannot use the free energy functional directly to derive any a priori bound for the solution which is not considered in the literature before. We discuss this issue in [23] in detail. One may summarize those existence and nonexistence results for the whole space case as follows:

Theorem $1.2([23],[17])$. Let $\lambda=0$ in (1.2). Suppose $u_{0} \in L^{1}\left(\mathbb{R}^{2}\right) \cap L^{2}\left(\mathbb{R}^{2}\right)$ is nonnegative everywhere.

(1) Then under the condition

$$
\int_{\mathbb{R}^{2}} u_{0}(x) d x<8 \pi
$$

the positive solution to (1.2) exists globally in time. Namely $(u, \psi) \in C\left([0, \infty) ;\left(L^{2} \cap L^{1}\right) \times\right.$ $\left.\dot{W}^{1, \infty}\right) \cap C^{1}\left((0, \infty) ; H^{2} \times \dot{W}^{2,1}\right)$ and for all $T>0$ there exists a finite constant $C=C(T)$ such that

$$
\int_{\mathbb{R}^{2}}\{(1+u(t)) \log (1+u(t))-u(t)\} d x \leq C(T), \quad t \in[0, T] .
$$

(2) On the other hand, if the positive initial data satisfies

$$
\int_{\mathbb{R}^{2}} u_{0}(x) d x>8 \pi
$$

then the solution does not exists globally. Namely it blows up in finite time.

Note that we do not assume the weight condition on the initial data both for the global existence and finite time blow up. The threshold case $\left\|u_{0}\right\|_{1}=8 \pi$ was considered recently by Biler-Karch-Laurençot-Nadzieja [5] for the radially symmetric case. 
1.2. Survey for degenerate case. The second problem we would like consider here is the degenerate version of the modified model of the Keller-Segel system

$$
\left\{\begin{array}{l}
\partial_{t} u-\Delta u^{\alpha}+\nabla \cdot(u \nabla \psi)=0, \quad x \in \mathbb{R}^{n}, t>0, \\
-\Delta \psi+\lambda \psi=u, \quad x \in \mathbb{R}^{n}, t>0, \\
u(x, 0)=u_{0}(x), \quad x \in \mathbb{R}^{n},
\end{array}\right.
$$

where $\alpha \geq 1$ and $\lambda>0$. An analogous variant of the semiconductor system like (1.3) is also our motivation. In that case, the nonlinear interaction of the stable sign is chosen.

The striking difference between the semilinear system (1.2) and the degenerate case (1.9) is that the equation essentially includes the hyperbolic structure in it and the finite propagation of the support of the solution may occur. If the solution is strictly positive, the solution is considered similarly as in the semilinear case. As is mentioned for the semilinear case, there exists a finite time blow up solution for a certain initial data and analogously the finite time blow up possibly occurs for the degenerate case. More precisely, when the data is positive and has the large initial value in the sense of $L^{1}$, then the solution for the modified version of the Keller-Segel system blows up in finite time ([20], [1]) when $n=2$ and for higher dimensional cases, the condition is getting weaker since the system is less stable. For the degenerate case, we expect an analogous situation.

If there is a point where the solution vanishes, the equation is essentially degenerate, and therefore the notion of a weak solution is required.

Definition. Let $\alpha \geq 1$. Given $u_{0} \in L^{1}\left(\mathbb{R}^{n}\right) \cap L^{\alpha}\left(\mathbb{R}^{n}\right)$ with $u_{0}(x) \geq 0$ for $x \in \mathbb{R}^{n}$, we call $(u(t, x), \psi(t, x))$ a weak solution of the system (1.9) if there exists $T>0$ such that

i) $u(t, x) \geq 0$ for any $(t, x) \in[0, T) \times \mathbb{R}^{n}$,

ii) $u \in C\left(\mathbb{R}^{n} \times[0, T)\right)$ with $\nabla u^{\alpha} \in L^{2}\left(\mathbb{R}^{n} \times[0, T)\right)$,

iii) For arbitrary test function $\phi \in C^{1,1}\left(\mathbb{R}^{n} \times[0, T)\right)$,

$$
\begin{aligned}
& \int_{\mathbb{R}^{n}} u(t) \phi(t) d x-\int_{\mathbb{R}^{n}} u_{0} \phi(0) d x \\
&=\int_{t_{0}}^{t_{1}} \int_{\mathbb{R}^{N}}\left(u(\tau) \partial_{t} \phi(\tau)-\nabla u^{\alpha}(\tau) \cdot \nabla \phi(\tau)+u(\tau) \nabla \psi(\tau) \cdot \nabla \phi(\tau)\right) d x d \tau
\end{aligned}
$$

for $0 \leq t \leq T$, where $\psi=E_{n} * u$ and $E_{n}(\cdot)$ is the fundamental solution of $-\Delta+\lambda$ in $\mathbb{R}^{n}$.

The difference between the degenerate case and semilinear case appears where the solution vanishes. In this regard, it is important to show the finite propagation of the support.

The existence of the weak solution is obtained by an application of the standard theory of parabolic equations. Since the equation neither satisfies the comparison principle of solutions nor has the semigroup representation as was possible for the semilinear case, the proof of the existence requires some approximation procedures involving the parabolic regularity theory. The following result due to Sugiyama [33] is one of the explicit examples (see also [35] for more general setting).

Theorem 1.3 ([33], local existence). For $\alpha>1$, there exists $T>0$ and a weak solution $(u, \psi)$ of the degenerate Keller-Segel system (1.9) for $u_{0} \in L^{1}\left(\mathbb{R}^{n}\right) \cap L^{\alpha}\left(\mathbb{R}^{n}\right)$. 
The exponent $\alpha=2-\frac{2}{n}$ corresponds to the Fujita exponent for the semilinear and quasilinear parabolic equation of the following type (cf. Fujita [10]):

$$
\left\{\begin{array}{c}
\partial_{t} u-\Delta u^{\alpha}=u^{p}, \quad x \in \mathbb{R}^{n}, t>0, \\
u(x, 0)=u_{0}(x), \quad x \in \mathbb{R}^{n},
\end{array}\right.
$$

where $\alpha \geq 0$. The exponent $p=\alpha+\frac{2}{n}=1+\frac{\sigma}{n}$ with $\sigma=\alpha(n-2)+2$ is a threshold for the global existence and finite time blow up for small data solutions. As in the semilinear case, we give the classification of the global existence and finite time blow up of the degenerate Keller-Segel equation. More specifically, if the exponent satisfies the other condition, then the solution blows up in a finite time for large initial data. This is essentially shown by Biler-Nadzieja-Stańczy [6] and Sugiyama [33].

Theorem 1.4 ([33], global existence). Let $\lambda=1$. For $\alpha>1$, let $(u, \psi)$ be a weak solution of the degenerate Keller-Segel system (1.9) for $u_{0} \geq 0$ obtained in the above Proposition.

(1) Let $n \geq 2$. If $\alpha>2-\frac{2}{n}$, then the solution exists globally in time. Moreover the weak solution satisfies the uniform estimate

$$
\|u(t)\|_{\infty} \leq C\left(\left\|u_{0}\right\|_{1},\left\|u_{0}\right\|_{\infty},\left\|\Lambda^{-1} u_{0}\right\|_{2}\right)
$$

for each $t \in[0, \infty)$.

(2) Let $n \geq 3$. If $\alpha \leq 2-\frac{2}{n}$ and the initial data is small in the following sense: there exists a constant $C>0$ such that

$$
\left\|u_{0}\right\|_{1} \leq C\left(\left\|E_{n}\right\|_{L_{w}^{\frac{n}{n-2}}}\right)
$$

where $E_{n}$ is the fundamental solution of $-\Delta+1$ in $\mathbb{R}^{n}$, then the solution exists globally in time, and moreover it satisfies the uniform estimate.

TheOrem 1.5 ([6], [33], finite time blow up). Let $(u, \psi)$ be a weak solution of the degenerate Keller-Segel system (1.9) with $\lambda=1$ for $u_{0} \geq 0$ obtained in the above Proposition. Assume that $n \geq 3$ and $\alpha \leq 2-\frac{2}{n}$, and the initial data $u_{0} \in L^{1}\left(\mathbb{R}^{n}\right) \cap L^{\alpha}\left(\mathbb{R}^{n}\right)$ with $|x|^{2} u_{0} \in L^{1}\left(\mathbb{R}^{n}\right)$ satisfies the following condition:

$$
W(0) \equiv \frac{1}{\alpha-1}\left\|u_{0}\right\|_{\alpha}^{\alpha}-\frac{1}{2}\left\|\Lambda^{-1} u_{0}\right\|_{2}^{2}<0,
$$

where $\Lambda=(-\Delta+1)^{1 / 2}$ is the Bessel potential in $\mathbb{R}^{n}$. Then the weak solution does not exist globally in time. Namely, there exists $T_{m}<\infty$ such that for some initial data $u_{0}$ the weak solution blows up in a finite time $T_{m}$ in the following sense:

$$
\limsup _{t \rightarrow T_{m}}\|u(t)\|_{p}=\infty
$$

for all $p \in[\alpha, \infty]$.

Remark. For the initial data satisfying the condition in Theorem 1.5, the $L^{1}$ norm of the data $u_{0}$ is naturally large. Especially for the critical case, $\alpha=2-\frac{2}{n}$, the data has to have large $L^{1}$ norm.

For the proof of the local existence of the weak solution, one may adopt the argument found in [35] and standard theory of degenerate parabolic systems (as in the theory of $p$ Laplace heat flow). In fact, the global existence result for the system is heavily depending 
on the a priori bound for the approximated solutions. One may find a priori bound by the argument in Theorem 1.4.

For bounded domain $\Omega$, the analogous blowing up problem is considered by BilerNadzieja-Stańczy [6]. They showed the nonexistence of the solution in the bounded domain $\Omega \subset \mathbb{R}^{n}$ for the Dirichlet boundary condition and the Neumann boundary condition. In those settings, the weighted density $\int_{\Omega}|x|^{2} u(t) d x$ can always make sense and the proof is rather simpler. The corresponding result for the Cauchy problem is also considered by Sugiyama [34].

For the semilinear case, $\alpha=1$, it has already been proved that $n=2$ is the critical case $\alpha=1=2-\frac{2}{n}$, and the solution may blow up in finite time for the large initial data. Our theorem is a natural extension of those semilinear cases. In particular, the case of the Cauchy problem, the threshold of the global existence and finite time blowing up of the solution is determined by the size of the $L^{1}$ norm of the initial data. Especially the semilinear critical case, the threshold number $8 \pi$ is connected with the best possible constant of the isoperimetric inequality via the Trudinger-Moser type inequality ([25]). The similar result can be also obtained by using the improved Brezis-Merle type inequality (Nagai-Ogawa [23]). For the degenerate case this corresponds to the best possible constant of the Hardy-Littlewood-Sobolev inequality (see for further discussions [34]).

The crucial part of the proof is to show the a priori bound for the weak solution in time globally. It is well understood that the solution of the semilinear equation (1.2) satisfies the following conservation laws in a formal way:

$$
\int_{\mathbb{R}^{n}} u(t) d x=\int_{\mathbb{R}^{n}} u_{0} d x, \quad W(t)+\int_{0}^{t} \int_{\mathbb{R}^{n}} u|K(u, \psi)|^{2} d x d t=W(0),
$$

with

$$
\begin{aligned}
& W(t) \equiv \int_{\mathbb{R}^{n}}(1+u(t)) \log (1+u(t)) d x-\int_{\mathbb{R}^{n}} u(t) v(t) d x+\frac{1}{2}\left(\|\nabla \psi(t)\|_{2}^{2}+\|\psi(t)\|_{2}^{2}\right), \\
& K(u(t), \psi(t)) \equiv \nabla(\log (1+u(t))-\psi) .
\end{aligned}
$$

The global existence part of the weak solution of the degenerate system in the above theorems depends essentially on the corresponding conservation laws of the quasilinear case (cf. for the semilinear case [3]).

Finally, we discuss the asymptotic behavior of the global small solution when the degeneracy order is less than the critical case.

We denote the weighted Lebesgue space $\left\{f \in L^{p}\left(\mathbb{R}^{n}\right):|\cdot|^{a} f(\cdot) \in L^{p}\left(\mathbb{R}^{n}\right)\right\}$ by $L_{a}^{p}\left(\mathbb{R}^{n}\right)$.

TheOREM 1.6 (Decay of solution). Let $1<\alpha \leq 2-\frac{2}{n}$ and assume that $u_{0} \in L_{2}^{1}\left(\mathbb{R}^{n}\right) \cap$ $L^{\infty}\left(\mathbb{R}^{n}\right)$. Then the corresponding global weak solution $u(t, x)$ of $(1.9)$ satisfies the following asymptotic behavior:

(1) For $1<\alpha \leq 2-\frac{2}{n}$, if $\left\|u_{0}\right\|_{1} \leq C_{n}$ for some absolute constant $C_{n}>0$, then

$$
\|u(t)\|_{p} \leq C(1+\sigma t)^{-\frac{n}{\sigma}\left(1-\frac{1}{p}\right)},
$$

where $C$ depends only on $u_{0}$ and $n$.

(2) For $1<\alpha<2-\frac{2}{n}$, assume that the initial data is small in the $L^{1}$ sense. Then for 
$M=\left\|u_{0}\right\|_{1}$ and for some $\nu>0$,

$$
\|u(t)-U(t)\|_{1} \leq C(1+\sigma t)^{-\nu},
$$

where $U(t)$ is the Barenblatt solution given by

$$
U(t)=(1+\sigma t)^{-n / \sigma}\left(A-\frac{\alpha-1}{2 \alpha} \frac{|x|^{2}}{(1+\sigma t)^{2 / \sigma}}\right)_{+}^{1 /(\alpha-1)},
$$

and $A>0$ is a constant such that $\|U(t)\|_{1}=M, \sigma=n(\alpha-1)+2$. In particular, the solution $u(t)$ satisfies the uniform decay estimate (1.11).

The semilinear version of the above asymptotic result has been obtained by several authors (see for example, Biler-Dolbeault [3] and Nagai-Syukuinn-Umesako [26] for the case (1.2)). The asymptotic profile including the critical case is shown by LuckhausSugiyama [18] in a slightly weaker way (see also [33]). The proof of the above asymptotic behavior depends on the behavior of the current functional. The method developed by Carrillo-Toscani [8] for the Fokker-Planck equation can be applied in our case. There is a variational formulation of the stationary solution of the porous medium equation (see Otto [29]) and the proof is based on this fact. Under the self-similar scaling (cf. Giga-Kohn [11], Biler-Dolbeault [3]) with new scaled variables $\left(t^{\prime}, x^{\prime}\right)$

$$
\left\{\begin{array}{l}
t^{\prime}=\frac{1}{\sigma} \log (1+\sigma t) \\
x^{\prime}=x /(1+\sigma t)^{1 / \sigma}
\end{array}\right.
$$

the rescaled equation is of the form

$$
\left\{\begin{array}{l}
\partial_{t^{\prime}} v-\operatorname{div} x_{x^{\prime}}\left(\nabla_{x^{\prime}} v^{\alpha}+x^{\prime} v-e^{-\kappa t^{\prime}} v \nabla_{x^{\prime}} \phi\right)=0, \quad t^{\prime}>0, x^{\prime} \in \mathbb{R}^{n}, \\
-e^{-2 t^{\prime}} \Delta_{x^{\prime}} \phi+\lambda \phi=v \\
v\left(0, x^{\prime}\right)=u_{0}\left(x^{\prime}\right)
\end{array}\right.
$$

with $\kappa=n+2-\sigma=n(2-\alpha)$, where

$$
\left\{\begin{array}{l}
v\left(t^{\prime}, x^{\prime}\right) \equiv e^{n t^{\prime}} u\left(\frac{1}{\sigma}\left(e^{\sigma t^{\prime}}-1\right), x^{\prime} e^{t^{\prime}}\right) \\
\phi\left(t^{\prime}, x^{\prime}\right) \equiv e^{n t^{\prime}} \psi\left(\frac{1}{\sigma}\left(e^{\sigma t^{\prime}}-1\right), x^{\prime} e^{t^{\prime}}\right)
\end{array}\right.
$$

The scaled equation (1.12) also has an analogous entropy. Setting

$$
\begin{aligned}
& W_{s}(v, \phi)(t) \equiv \frac{1}{2} H(v(t))-\frac{1}{2} e^{-\kappa t} \int_{\mathbb{R}^{n}} v(t) \phi(t) d x \\
& H(v(t))=\frac{2}{\alpha-1} \int_{\mathbb{R}^{n}} v^{\alpha}(t) d x+\int_{\mathbb{R}^{n}}|x|^{2} v(t) d x \\
& K_{s}(x, v(t), \phi(t))=K(x, v, \phi) \equiv \nabla\left(\frac{\alpha}{\alpha-1} v^{\alpha-1}+\frac{1}{2}|x|^{2}-e^{-\kappa t} \phi\right),
\end{aligned}
$$

the following identities hold formally (Proposition 4.1):

$$
\begin{aligned}
& \int_{\mathbb{R}^{n}} v(t) d x=\int_{\mathbb{R}^{n}} u_{0}(x) d x \\
& W_{s}(t)+\int_{0}^{t}\left[\int_{\mathbb{R}^{n}} v|K(x, v, \phi)(\tau)|^{2} d x d \tau+e^{-(\kappa+2) \tau} \int_{\mathbb{R}^{n}}|\nabla \phi(\tau)|^{2} d x\right] d \tau=W_{s}(0) .
\end{aligned}
$$


Hence the decay of the solution follows from the analogous estimate for the global existence of the weak solution. The convergence to the limiting solution is obtained from computations of the second time derivative of the moment.

This paper is organized as follows. In the next section, we derive the above entropy and free energy bound formally. Based on this conserved quantities, we show the time a priori estimate for the global weak solution in section 2 . In section 3 , we give the formal blow up proof. The last two sections 4 and 5 are devoted to the proof of the decay of the solution.

\section{Free energy estimate and uniform a priori bound}

2.1. Conserved quantities. We start with the following lemma for the conservation law and the entropy functional:

Lemma 2.1. Let $(u, \psi)$ be a weak solution of (1.9). Then

$$
\begin{aligned}
& \int_{\mathbb{R}^{n}} u(t) d x=\int_{\mathbb{R}^{n}} u_{0}(x) d x \\
& W(t)+\int_{0}^{t} \int_{\mathbb{R}^{n}} u|K(u, \psi)|^{2} d x d t \leq W(0)
\end{aligned}
$$

with

$$
\begin{aligned}
& W(t) \equiv \frac{1}{\alpha-1}\|u(t)\|_{\alpha}^{\alpha}-\frac{1}{2} \int_{\mathbb{R}^{n}} u(t) \psi(t) d x, \\
& K(u(t), \psi(t)) \equiv \nabla\left(\frac{\alpha}{\alpha-1} u^{\alpha-1}-\psi\right) .
\end{aligned}
$$

Proof. For completeness, we show the formal proof of those conservation laws. Multiplying (1.9) by $\frac{\alpha}{\alpha-1} u^{\alpha-1}-\psi$ and integrating by parts, we see

$$
\begin{aligned}
\int_{\mathbb{R}^{n}} \partial_{t} u\left(\frac{\alpha}{\alpha-1} u^{\alpha-1}-\psi\right) d x & =-\int_{\mathbb{R}^{n}}\left(\nabla u^{\alpha}-u \cdot \nabla \psi\right) \cdot \nabla\left(\frac{\alpha}{\alpha-1} u^{\alpha-1}-\psi\right) d x \\
& =-\int_{\mathbb{R}^{n}} u\left|\nabla\left(\frac{\alpha}{\alpha-1} u^{\alpha-1}-\psi\right)\right|^{2} d x
\end{aligned}
$$

From the second equation,

$$
\int_{\mathbb{R}^{n}} u \cdot \partial_{t} \psi d x=\frac{1}{2} \frac{d}{d t} \int_{\mathbb{R}^{n}}\left(|\nabla \psi|^{2}+|\psi|^{2}\right) d x .
$$

Thus, the left hand side of (2.4) is

$$
\frac{d}{d t}\left(\frac{1}{\alpha-1}\|u(t)\|_{\alpha}^{\alpha}-\int_{\mathbb{R}^{n}} u \psi d x+\frac{1}{2} \int_{\mathbb{R}^{n}}\left(|\nabla \psi|^{2}+|\psi|^{2}\right) d x\right) .
$$

Combining (2.4) and (2.5) we get

$$
\frac{d}{d t} W(t)+\int_{\mathbb{R}^{n}} u\left|\nabla\left(\frac{\alpha}{\alpha-1} u^{\alpha-1}-\psi\right)\right|^{2} d x=0 .
$$

Integrating in time both sides of (2.6), we obtain the desired estimate. The rigorous justification requires some regularizing argument for the equation in order to escape the degeneracy (see [34]). 
2.2. Uniform boundedness. We show only the a priori estimates for the global existence of the weak solution. The local existence theorem requires some approximation procedures but we do not discuss the details.

Under the condition $\alpha>2-\frac{2}{n}$, we show the uniform boundedness of the solution $(u, \psi)$. To see this, the a priori bound for $L^{\alpha}$ is essential. We begin with the following auxiliary lemma:

Lemma 2.2. Let $E_{n}$ be the fundamental solution of $(-\Delta+1)$ in $\mathbb{R}^{n}$. Then for $u \in$ $L^{1}\left(\mathbb{R}^{n}\right) \cap L^{\alpha}\left(\mathbb{R}^{n}\right)$, and $\psi=E_{n} u$ the solution of the second equation of the system (1.9), we have

$$
\int_{\mathbb{R}^{n}} u \psi d x=\frac{1}{2}\left(\|\nabla \psi\|_{2}^{2}+\|\psi\|_{2}^{2}\right) \leq\left\|E_{n}\right\|_{L_{w}^{\frac{n}{n-2}}}\|u\|_{1}^{1-\gamma}\|u\|_{\alpha}^{1+\gamma},
$$

where $\gamma=\frac{\alpha(n-2)}{n(\alpha-1)}-1<\alpha-1$.

Proof. The first identity in (2.7) is obtained directly from the second equation. By the Hölder inequality

$$
\int_{\mathbb{R}^{n}} u \psi d x \leq\|u\|_{r}\|\psi\|_{r^{\prime}} \quad \text { for } \frac{1}{r}+\frac{1}{r^{\prime}}=1
$$

while

$$
\|u(t)\|_{r} \leq\|u\|_{1}^{1-\gamma}\|u\|_{\alpha}^{\gamma}
$$

under

$$
\frac{1}{r}=1-\gamma+\frac{\gamma}{\alpha}
$$

and by the Hausdorff-Young inequality,

$$
\|\psi\|_{r^{\prime}} \leq\left\|E_{n}\right\|_{L_{w}^{\frac{n}{n-2}}}\|u\|_{\alpha}
$$

with

$$
\frac{1}{r^{\prime}}=\frac{n-2}{n}+\frac{1}{\alpha}-1
$$

If we wish to choose $\gamma+1<\alpha$, then by

$$
\gamma\left(1-\frac{1}{\alpha}\right)=1-\frac{1}{r}=\frac{n-2}{n}+\frac{1}{\alpha}-1,
$$

we see that

$$
\alpha\left(1-\frac{1}{\alpha}\right)>(\gamma+1)\left(1-\frac{1}{\alpha}\right)=\frac{n-2}{n}
$$

This relation yields

$$
\alpha>2-\frac{2}{n},
$$

and this condition gives the uniform boundedness of the $L^{\alpha}$ norm of the solution. 
Proposition 2.3. (1) Let $\alpha>2-\frac{2}{n}$. Then we have

$$
\|u(t)\|_{\alpha}^{\alpha}+\frac{1}{2}\left(\|\nabla \psi(t)\|_{2}^{2}+\|\psi(t)\|_{2}^{2}\right) \leq C\left(W(0)+C_{n}\left\|u_{0}\right\|_{1}^{\frac{\alpha(\gamma+1)}{\alpha+\gamma-1}}\right)
$$

for all $t \in[0, \infty)$, where $\gamma<\alpha-1$. In particular,

$$
\left\|u_{0}\right\|_{\alpha}^{\alpha} \leq C\left(W(0)+C_{n}\left\|u_{0}\right\|_{1}^{\frac{\alpha(\gamma+1)}{\alpha+\gamma-1}}\right)
$$

(2) Let $1<\alpha \leq 2-\frac{2}{n}$. Then there exists a constant $C=C_{n}$ which depends only on $n$ such that for the weak solution satisfying $\left\|u_{0}\right\|_{1} \leq C_{n}$, we have

$$
\|u(t)\|_{\alpha}^{\alpha} \leq C\left(n,\left\|u_{0}\right\|_{1}, W(0)\right)
$$

for all $t \in[0, \infty)$.

Proof. By the entropy bound (2.2), it suffices to show that

$$
\int_{\mathbb{R}^{n}} u(t) \psi(t) d x
$$

is controlled by $\|u(t)\|_{\alpha}^{\alpha}$ and $W(0)$. To see this we see by Lemma 2.2 that under the condition $\alpha>2-\frac{2}{n}$,

$$
\int_{\mathbb{R}^{n}} u(t) \psi(t) d x \leq C\|u(t)\|_{1}^{1-\gamma}\|u(t)\|_{\alpha}^{1+\gamma} \leq \varepsilon\|u(t)\|_{\alpha}^{\alpha}+C_{\varepsilon}\left\|u_{0}\right\|_{1}^{\frac{\alpha(1-\gamma)}{\alpha-(\gamma+1)}},
$$

and the desired estimate follows from $(2.9), L^{1}$ conservation law and the entropy bound $W(t) \leq W(0)$.

For the case $1<\alpha \leq 2-\frac{2}{n}$, we again use Lemma 2.2 and the entropy bound (2.2), to see that

$$
\frac{2}{\alpha-1}\|u(t)\|_{\alpha}^{\alpha} \leq W(0)+\left\|E_{n}\right\|_{L_{w}^{n /(n-2)}}\left\|u_{0}\right\|_{1}^{1-\gamma}\|u(t)\|_{\alpha}^{\gamma+1} .
$$

If $\alpha=2-\frac{2}{n}$, then $\gamma+1=\alpha$ and the smallness condition

$$
\left\|u_{0}\right\|_{1}^{1-\gamma}<\frac{2}{\alpha-1}\left\|E_{n}\right\|_{L_{w}^{n /(n-2)}}^{-1}
$$

directly gives the uniform boundedness of $\|u(t)\|_{\alpha}$. For $1<\alpha<2-\frac{2}{n}$, there exists a constant $C_{0}$ which is determined by $W(0), \alpha, n$ and $\left\|E_{n}\right\|_{L_{w}^{n /(n-2)}}$ such that for $\left\|u_{0}\right\|_{1}$ $\leq C_{0}$ we also see that

$$
\|u(t)\|_{\alpha}^{\alpha} \leq C\left(n, \alpha,\left\|u_{0}\right\|_{1}, W(0)\right)
$$

uniformly in $t$.

REMARK. The exponent of $L^{1}$ norm of the right hand side of (2.9) satisfies

$$
\frac{\alpha(1-\gamma)}{\alpha-(\gamma+1)} \geq 2
$$

THEOREM 2.4. Let $n \geq 2$ and suppose that $\alpha>1$. Then, under the condition that $\|u(t)\|_{\alpha}$ is uniformly bounded in $t$, we have for any $t>0$,

$$
\|u(t)\|_{\infty}+\|\psi(t)\|_{\infty} \leq C\left(W(0),\left\|u_{0}\right\|_{1},\left\|u_{0}\right\|_{\infty}, \alpha, n\right),
$$

hence the weak solution exists globally. 
Proof. Firstly we observe that for some $r_{0}>n$, we have the uniform bounded estimate for $\|u(t)\|_{r_{0}}$ by Proposition 2.3. We apply the standard parabolic estimates and we see for any $r>\alpha$ that

$$
\frac{d}{d t}\|u(t)\|_{r}^{r}+\frac{2 r}{\alpha+r-1}\left\|\nabla u^{\gamma}(t)\right\|_{2}^{2}=\int_{\mathbb{R}^{n}} \nabla u^{r}(t) \cdot \nabla \psi(t) d x \leq\|u(t)\|_{r+1}^{r+1},
$$

where $\gamma=\frac{1}{2}(\alpha+r-1)$. Now we invoke the Gagliardo-Nirenberg interpolation inequality

$$
\begin{aligned}
& \|f\|_{(r+1) / \gamma} \leq C\|f\|_{r / \chi \gamma}^{1-\sigma}\|\nabla f\|_{2}^{\sigma}, \\
& \quad \frac{\gamma}{r+1}=\frac{\chi \gamma(1-\sigma)}{r}+\sigma\left(\frac{1}{2}-\frac{1}{n}\right)
\end{aligned}
$$

for some $\chi>1$. It follows by substituting $f=u^{\gamma}(t)$ that

$$
\|u(t)\|_{r+1}^{r+1} \leq C\|u(t)\|_{r / \chi}^{(r+1)(1-\sigma)}\left\|\nabla u^{\gamma}(t)\right\|_{2}^{\sigma(r+1) / \gamma} .
$$

If we assume that $\sigma(r+1) / \gamma<2$ which is assured under the condition

$$
2-\frac{2 r}{\chi n}<\alpha
$$

we have

$$
\|u(t)\|_{r+1}^{r+1} \leq C\|u(t)\|_{r / \chi}^{\beta}+\left\|\nabla u^{\gamma}(t)\right\|_{2}^{2}
$$

with

$$
\beta=(1-\sigma)(r+1)\left(1-\frac{2 \gamma}{\sigma(r+1)}\right)^{-1}
$$

Similarly, we see that

$$
\begin{aligned}
\|u(t)\|_{r}^{r} & \leq C\|u(t)\|_{1}^{r(1-\mu)}\left\|\nabla u^{\gamma}(t)\right\|_{2}^{r \mu / \gamma} \\
& \leq C\|u(t)\|_{1}^{\delta}+\left\|\nabla u^{\gamma}(t)\right\|_{2}^{2}
\end{aligned}
$$

where $r \mu / \gamma<2$ under $1-\frac{r}{n}<\alpha$ and

$$
\begin{aligned}
\delta & =(1-\mu) r\left(1-\frac{r \mu}{2 \gamma}\right)^{-1} \\
& =\frac{(1-\mu)(1+(\alpha-1) / r)}{1-\mu+(\alpha-1) / r} r \\
& =\frac{1-\mu+(1-\mu)(\alpha-1) / r}{1-\mu+(\alpha-1) / r} r<r,
\end{aligned}
$$

since $(1-\mu)(\alpha-1) / r<(\alpha-1) / r$. Thus, combining (2.10), (2.13) and (2.14), we obtain

$$
\frac{d}{d t}\|u(t)\|_{r}^{r}+C_{0}\|u(t)\|_{r}^{r} \leq C\|u(t)\|_{r / \chi}^{\beta}+C\|u(t)\|_{1}^{\delta}
$$

with

$$
\beta=(1-\sigma)(1+r)\left(1-\frac{\sigma(r+1)}{2 \gamma}\right)^{-1} .
$$

Let us first choose $\chi=r / \alpha>1$. Then we see from (2.15) that

$$
\frac{d}{d t}\|u(t)\|_{r_{0}}^{r_{0}}+C_{0}\|u(t)\|_{r_{0}}^{r_{0}} \leq C\|u(t)\|_{\alpha}^{\beta}+C\|u(t)\|_{1}^{\delta},
$$


under the condition that $2-\frac{4}{n+2}<\alpha$. Since by the assumption, the right hand side is uniformly bounded in $t$, multiplying by $e^{C_{0} t}$ both sides of the inequality, it follows that

$$
\begin{aligned}
\|u(t)\|_{r_{0}}^{r_{0}} & \leq\left\|u_{0}\right\|_{r_{0}}^{r_{0}}+\left(C \sup _{t \in[0, T]}\|u(t)\|_{\alpha}^{\beta}+C\left\|u_{0}\right\|_{1}^{\delta}\right) \int_{0}^{t} e^{-C_{0}(t-s)} d s \\
& \leq\left\|u_{0}\right\|_{r_{0}}^{r_{0}}+C\left\|u_{0}\right\|_{1}^{\delta}+C \sup _{t \in[0, T]}\|u(t)\|_{\alpha}^{\beta} .
\end{aligned}
$$

The above estimate combined with the Sobolev inequality and the elliptic estimates imply

$$
\|\nabla \psi(t)\|_{\infty} \leq C\|u(t)\|_{r_{0}}
$$

for some $r_{0}>n$, where $C$ is independent of $t$, implies the uniform bound for $\|\nabla \psi(t)\|_{\infty}$.

Now we show for the general case $r \in\left[r_{0}, \infty\right]$. Starting from the $L^{r}$ inequality $(2.10)$

$$
\begin{aligned}
\frac{d}{d t}\|u(t)\|_{r}^{r}+\frac{2 r}{\alpha+r-1}\left\|\nabla u^{\gamma}(t)\right\|_{2}^{2} & \leq\|\nabla \psi(t)\|_{\infty} \int_{\mathbb{R}^{n}}\left|\nabla u^{r}\right| d x \\
& \leq \frac{2 r\|\nabla \psi\|_{\infty}}{r+\alpha-1}\|u(t)\|_{r-\alpha+1}^{(r-\alpha+1) / 2}\left\|\nabla u^{\gamma}\right\|_{2},
\end{aligned}
$$

where $\gamma=\frac{1}{2}(\alpha+r-1)$. Analogous to (2.11), the Gagliardo-Nirenberg interpolation inequality

$$
\begin{aligned}
& \|f\|_{(r+1-\alpha) / \gamma} \leq C\|f\|_{r / \chi \gamma}^{1-\sigma}\|\nabla f\|_{2}^{\sigma}, \\
& \frac{\gamma}{r+1-\alpha}=\frac{\chi \gamma(1-\sigma)}{r}+\sigma\left(\frac{1}{2}-\frac{1}{n}\right)
\end{aligned}
$$

holds for some $\chi>1$. It then follows by substituting $f=u^{\gamma}(t)$ that

$$
\|u(t)\|_{r+1-\alpha}^{r+1-\alpha} \leq C\|u(t)\|_{r / \chi}^{(r+1-\alpha)(1-\sigma)}\left\|\nabla u^{\gamma}(t)\right\|_{2}^{\sigma(r+1-\alpha) / \gamma} .
$$

If we assume that $\sigma(r+1-\alpha) / \gamma<2$ which is assured under the condition

$$
1-\frac{r}{n}<\alpha
$$

we have

$$
\|u(t)\|_{r+1-\alpha}^{r+1-\alpha} \leq C\|u(t)\|_{r / 2}^{\beta}+\left\|\nabla u^{\gamma}(t)\right\|_{2}^{2}
$$

where

$$
\begin{aligned}
\beta & =(1-\sigma)(1+r-\alpha)\left(1-\frac{\sigma(r+1-\alpha)}{2 \gamma}\right)^{-1} \\
& =\frac{2 \gamma(1-\sigma)}{2 \gamma /(r+1-\alpha)-\sigma} \\
& =r \frac{1-\sigma+(1-\sigma)(\alpha-1) / r}{1-\sigma+2(\alpha-1) /(r+1-\alpha)}<r
\end{aligned}
$$

Thus we again use (2.10), (2.15) to find that

$$
\frac{d}{d t}\|u(t)\|_{r}^{r}+C_{0}\|u(t)\|_{r}^{r} \leq C\|u(t)\|_{r / \chi}^{\beta}+C\|u(t)\|_{1}^{\delta}
$$

under the condition $2-\frac{4}{n+2}<\alpha$. Note that all the constants appearing in the above inequality depend on $r$ but they can be chosen uniformly bounded as $r \rightarrow \infty$. Multiplying 
by $e^{C_{0} t}$ both sides of the inequality, it follows that

$$
\|u(t)\|_{r}^{r} \leq\left\|u_{0}\right\|_{r}^{r}+\left(C \sup _{t \in[0, T]}\|u(t)\|_{r / \chi}^{\beta}+C\left\|u_{0}\right\|_{1}^{\delta}\right) \int_{0}^{t} e^{-C_{0}(t-s)} d s .
$$

For sufficiently large $r>n$, we see that

$$
\|u(t)\|_{r}+M \leq C^{1 / r}\left(M+\sup _{t \in[0, T]}\|u(t)\|_{r / \chi}\right)
$$

for all $t \in[0, T]$, where $M=\max \left(\left\|u_{0}\right\|_{1},\left\|u_{0}\right\|_{\infty}\right)$. Now choosing $r=\chi^{m}$, we see by Nash-Moser iteration argument that

$$
\|u(t)\|_{\infty} \leq C^{c \sum_{m} \chi^{-m}}\left(M+\sup _{t \in[0, T]}\|u(t)\|_{r_{0}}\right) .
$$

This combined with the estimate (2.16) yields the desired uniform estimate under the uniform bound of $\|u(t)\|_{\alpha}$.

For the case $1<\alpha \leq 2-\frac{4}{n+2}$, we return to the estimate (2.15):

$$
\frac{d}{d t}\|u(t)\|_{r}^{r}+C_{0}\|u(t)\|_{r}^{r} \leq C\|u(t)\|_{r / \chi}^{\beta}+C\|u(t)\|_{1}^{\delta}
$$

with

$$
\beta=\frac{2 \gamma(1-\sigma)}{2 \gamma /(r+1)-\sigma}=r \frac{1-\sigma+(1-\sigma)(\alpha-1) / r}{1-\sigma+(\alpha-2) /(r+1)} \equiv r \mu_{r} .
$$

This yields an analogous estimate as in (2.2) with $\mu_{r}$ maybe larger than 1 . Let $r=r_{0}>n$ fixed and we choose $\chi>1$ properly so that by finite number of iterations,

$$
\|u(t)\|_{r_{0}} \leq C^{c \sum_{k=1}^{m} \chi^{-m}}\left(M+\sup _{t \in[0, T]}\|u(t)\|_{r_{0} / \chi^{m}}\right)^{c \prod_{k=1}^{m} \mu_{\chi^{k}}},
$$

where the exponent $r / \chi^{m}$ is in $[1, \alpha]$ and hence we have

$$
\|u(t)\|_{r_{0}} \leq C \text { independent of } t .
$$

The remainder of the argument is similar to the case $2-\frac{4}{n+2}<\alpha$.

Note that we can eliminate the initial restriction $\left\|u_{0}\right\|_{r}$ by the parabolic regularity argument. The regularity of $\psi(t)$ immediately follows from standard elliptic estimates for the second equation.

Lemma 2.5. Let $\alpha>1$. For any $f \in L^{\alpha}\left(\mathbb{R}^{n}\right)$ with $|x|^{2} u_{0}(x) \in L^{1}\left(\mathbb{R}^{n}\right)$, we have

$$
\|f\|_{1} \leq C\left(\int_{\mathbb{R}^{n}}|x|^{2}|f(x)| d x\right)^{1-\kappa}\|f\|_{\alpha}^{\kappa}
$$

where

$$
\kappa=\frac{2 \alpha}{\alpha(n+2)-n} .
$$

Proof. For simplicity we assume $f \geq 0$. The general case can be easily obtained by a simple modification. For some constants $a, b>0$, and $r>0$ to be chosen later, we see 
that

$$
\begin{aligned}
\int_{\mathbb{R}^{n}} f(x) d x & \leq \int_{B_{r}} f(x) d x+\int_{B_{r}^{c}} f(x) d x \\
& \leq a r^{n / \alpha^{\prime}}\left(\int_{B_{r}}|f(x)|^{\alpha} d x\right)^{1 / \alpha}+\frac{b}{r^{2}} \int_{B_{r}^{c}}|x|^{2} f(x) d x \\
& \leq a r^{n / \alpha^{\prime}}\|f\|_{\alpha}+\frac{b}{r^{2}} \int_{\mathbb{R}^{n}}|x|^{2} f(x) d x \\
& \equiv A r^{n(\alpha-1) / \alpha}+B r^{-2} \equiv f(r) .
\end{aligned}
$$

Then $f^{\prime}(r)=\frac{n}{\alpha} A r^{n / \alpha-1}-2 B r^{-3}=0$ gives

$$
r^{\frac{n}{\alpha^{\prime}}+2}=c \frac{B}{A} .
$$

Thus

$$
r=c\left(\frac{B}{A}\right)^{\frac{\alpha}{\alpha(n+2)-n}}
$$

and the desired inequality follows.

\section{Finite time blow up}

3.1. Dimension analysis. Let $\lambda>0$ and $\mu>0$ be scaling parameters. We introduce the following scaled solutions:

$$
\left\{\begin{array}{l}
u_{\lambda, \mu}=\lambda u(\mu x) \\
\psi_{\lambda, \mu}=\lambda \mu^{-2} \psi(\mu x)
\end{array}\right.
$$

A direct computation gives

LEMMA 3.1.

$$
\begin{aligned}
\left\|u_{\lambda, \mu}\right\|_{1} & =\lambda \mu^{-n}\|u\|_{1} \\
\left\|u_{\lambda}\right\|_{\alpha}^{\alpha} & =\lambda^{\alpha} \mu^{-n}\|u\|_{\alpha}^{\alpha} \\
\int_{\mathbb{R}^{n}} u_{\lambda} \psi_{\lambda} d x & =\lambda^{2}\langle\mu\rangle^{-(n+2)} \int_{\mathbb{R}^{n}} u \psi d x .
\end{aligned}
$$

Now we see that the initial entropy may be chosen negative (cf. [6]).

Lemma 3.2. Let $n \geq 2$ and $u \in L^{1}\left(\mathbb{R}^{n}\right) \cap L^{\alpha}\left(\mathbb{R}^{n}\right)$. For $\lambda, \mu>0$ we define the scaled function $u_{\lambda, \mu}=\lambda u(\mu x)$. Set $\left\|u_{\alpha, \mu}\right\|_{1}=A$, then for $\alpha<2-\frac{2}{n}$, by choosing $\lambda \gg 1$ large enough,

$$
W(0)=\frac{1}{\alpha-1}\left\|u_{\lambda, \mu}\right\|_{\alpha}^{\alpha}-\frac{1}{2} \int_{\mathbb{R}^{n}} u_{\lambda, \mu}(-\Delta+1)^{-1} u_{\lambda, \mu} d x<0 .
$$

If $\alpha=2-\frac{2}{n}$, then choosing A sufficiently large, we have the same conclusion.

Proof. By $\left\|u_{\lambda, \mu}\right\|_{1}=\lambda \mu^{-n}=A$, we have $\mu=(\lambda / A)^{1 / n}$. Then we have for the positive constants $B=\frac{\|u\|_{\alpha}^{\alpha}}{\alpha-1}, C=\frac{1}{2}\left\|\Lambda^{-1} u\right\|_{2}^{2}$, 


$$
\begin{aligned}
W\left(u_{\lambda, \mu}\right) & =\frac{1}{\alpha-1} \lambda^{\alpha} \mu^{-n}\|u\|_{\alpha}^{\alpha}-\frac{1}{2} \lambda^{2} \mu^{-n}\langle\mu\rangle^{-2} \int_{\mathbb{R}^{n}} u(-\Delta+1)^{-1} u d x \\
& =A B \lambda^{\alpha-1}-C \frac{\lambda^{2} A^{\frac{n+2}{n}}}{\lambda\left(A^{\frac{1}{n}}+\lambda^{\frac{1}{n}}\right)^{2}} \\
& =A \lambda^{\alpha-1}\left(B-\frac{\lambda^{2-\alpha} A^{\frac{2}{n}} C}{\left(A^{\frac{1}{n}}+\lambda^{\frac{1}{n}}\right)^{2}}\right) \\
& = \begin{cases}A \lambda^{\alpha-1}\left(B-A^{2 / n} C \lambda^{2-\frac{2}{n}-\alpha}\right), & \lambda>1, \\
A \lambda^{\alpha-1}\left(B-C \lambda^{2-\alpha}\right), & \lambda<1 .\end{cases}
\end{aligned}
$$

Hence if $\alpha<2-\frac{2}{n}$ then choosing $\lambda$ large, we have $W<0$. If $\alpha=2-\frac{2}{n}$, then choose $A=\|u\|_{1}$ sufficiently large, and $W<0$.

3.2. Virial law and blow up. In this section, we show the nonexistence of the weak solution and finite time blow up in a formal way. The argument is almost similar to that in [6] (cf. [28], [33]).

Lemma 3.3. Let $(u, \psi)$ be a weak solution of (1.9). Then

$$
\frac{d}{d t} \int_{\mathbb{R}^{n}}|x|^{2} u(t) d x=2 n\|u(t)\|_{\alpha}^{\alpha}+2 \int_{\mathbb{R}^{n}} x u(t) \cdot \nabla \psi(t) d x .
$$

Proof. Multiply the equation by $|x|^{2}$ and integrate this by parts.

Here we show a rough result on the finite time blow up.

Theorem 3.4 ([6], [33]). Let $n \geq 3$ and $1 \leq \alpha \leq 2-\frac{2}{n}$. Then for $u_{0} \in L^{1}\left(\mathbb{R}^{n}\right) \cap L^{\alpha}\left(\mathbb{R}^{n}\right)$ with $|x|^{2} u_{0}(x) \in L^{1}\left(\mathbb{R}^{n}\right)$ and

$$
W(0) \equiv \frac{1}{\alpha-1}\left\|u_{0}\right\|_{\alpha}^{\alpha}-\frac{1}{2}\left\|(-\Delta+1)^{-1 / 2} u_{0}\right\|_{2}^{2}<0
$$

the corresponding weak solution obtained in Proposition 1.3 blows up in a finite time.

Proof. The proof is essentially similar to that in [6]. We only give the formal calculation. First we see from Lemma 3.3 that

$$
\frac{d}{d t} \int_{\mathbb{R}^{n}}|x|^{2} u(t) d x=2 n\|u(t)\|_{\alpha}^{\alpha}+2 \int_{\mathbb{R}^{n}} x u(t) \cdot \nabla \psi(t) d x .
$$

Next we invoke the Pokhozaev identity for the second equation. We multiply the elliptic part of the system by the generator of the dilation $x \cdot \nabla \psi$ and integrate it by parts. Then

$$
\begin{aligned}
\int_{\mathbb{R}^{n}} x \cdot \nabla \psi(t) u(t) d x & =\int_{\mathbb{R}^{n}} \nabla_{i} \psi(t)\left(\delta_{i j} \nabla_{j} \psi(t)+x \cdot \nabla \nabla_{i} \psi(t)\right) d x+\frac{1}{2} \int_{\mathbb{R}^{n}} x \cdot \nabla|\psi(t)|^{2} d x \\
& =\left(1-\frac{n}{2}\right) \int_{\mathbb{R}^{n}}|\nabla \psi(t)|^{2} d x-\frac{n}{2} \int_{\mathbb{R}^{n}}|\psi(t)|^{2} d x \\
& =\left(1-\frac{n}{2}\right) \int_{\mathbb{R}^{n}} u(t) \psi(t) d x-\|\psi(t)\|_{2}^{2}
\end{aligned}
$$


Combining (3.6) and (3.7), we obtain

$$
\begin{aligned}
\frac{d}{d t} \int_{\mathbb{R}^{n}}|x|^{2} u(t) d x= & 2 n\|u(t)\|_{\alpha}^{\alpha}+(2-n) \int_{\mathbb{R}^{n}} u(t) \psi(t) d x-2\|\psi(t)\|_{2}^{2} \\
= & 2(n-2)\left(\frac{1}{\alpha-1}\|u(t)\|_{\alpha}^{\alpha}-\frac{1}{2} \int_{\mathbb{R}^{n}} u(t) \psi(t) d x\right) \\
& +\left(2 n-\frac{2(n-2)}{\alpha-1}\right)\|u(t)\|_{\alpha}^{\alpha}-2\|\psi\|_{2}^{2} \\
= & 2(n-2) W(t)+2 n\left(\frac{\alpha-2+\frac{2}{n}}{\alpha-1}\right)\|u(t)\|_{\alpha}^{\alpha}-2\|\psi\|_{2}^{2} .
\end{aligned}
$$

Hence, assuming $n \geq 3$ and $\alpha \leq 2-\frac{2}{n}$, it is possible to choose the initial data such that $W(0)<0$, and we see by Lemma 3.1 that

$$
\frac{d}{d t} \int_{\mathbb{R}^{n}}|x|^{2} u(t) d x \leq W(0)<0,
$$

which yields a contradiction within a finite time.

4. Time decay of small solutions. In this section, we consider the decay and asymptotic behavior of the global weak solution of the degenerate Keller-Segel system.

4.1. Rescaled equation. To avoid the confusion, we change the notation slightly

$$
\left\{\begin{array}{l}
\partial_{t} u-\Delta u^{\alpha}+\nabla(u \nabla \psi)=0, \quad x \in \mathbb{R}^{n}, t>0 \\
-\Delta \psi+\lambda \psi=u \quad x \in \mathbb{R}^{n}, t>0 \\
u(0, x)=u_{0}(x), \quad x \in \mathbb{R}^{n}
\end{array}\right.
$$

We introduce the new scaled variables $\left(t^{\prime}, x^{\prime}\right)$ as

$$
\left\{\begin{array}{l}
t^{\prime}=\frac{1}{\sigma} \log (1+\sigma t) \\
x^{\prime}=x /(1+\sigma t)^{1 / \sigma}
\end{array}\right.
$$

and introduce the new scaled unknown function $u\left(t^{\prime}, x^{\prime}\right)$. Concerning the presence of $\lambda>0$, we may choose the scaling that keeps the $\lambda$ term unchanged, namely

$$
\begin{aligned}
& u(t, x)=(1+\sigma t)^{-n / \sigma} v\left(\frac{1}{\sigma} \log (1+\sigma t), \frac{x}{(1+\sigma t)^{1 / \sigma}}\right), \\
& \psi(t, x)=(1+\sigma t)^{-n / \sigma} \phi\left(\frac{1}{\sigma} \log (1+\sigma t), \frac{x}{(1+\sigma t)^{1 / \sigma}}\right) .
\end{aligned}
$$

This may be written as

$$
\begin{aligned}
& v\left(t^{\prime}, x^{\prime}\right) \equiv e^{n t^{\prime}} u\left(\frac{1}{\sigma}\left(e^{\sigma t^{\prime}}-1\right), x^{\prime} e^{t^{\prime}}\right), \\
& \phi\left(t^{\prime}, x^{\prime}\right) \equiv e^{n t^{\prime}} \psi\left(\frac{1}{\sigma}\left(e^{\sigma t^{\prime}}-1\right), x^{\prime} e^{t^{\prime}}\right),
\end{aligned}
$$


and the resulting equation for $(v, \phi)$ follows by setting $\kappa=n+2-\sigma=n(2-\alpha)$,

$$
\left\{\begin{array}{l}
\partial_{t^{\prime}} v-\operatorname{div}_{x^{\prime}}\left(\nabla_{x^{\prime}} v^{\alpha}+x^{\prime} v-e^{-\kappa t^{\prime}} v \nabla_{x^{\prime}} \phi\right)=0, \quad t^{\prime}>0, x^{\prime} \in \mathbb{R}^{n}, \\
-e^{-2 t} \Delta_{x^{\prime}} \phi+\lambda \phi=v \\
v\left(0, x^{\prime}\right)=u_{0}\left(x^{\prime}\right)
\end{array}\right.
$$

In this case, the vanishing exponent can be found as before as $\alpha=2$ by $0=\sigma-n-2=$ $n(\alpha-2)$, and thus the subcritical case corresponds to $\alpha<2$. Hereafter we analyze the above rescaled equation (4.3) to get the asymptotic behavior of the solution.

The existence of the weak solution of (4.3) can be proven similarly as for the original equation. Indeed, the scaling does not change any analytic feature of the original weak solution so that the solution can be obtained from the weak solution of (1.9). Namely, we again consider the nonnegative weak solution $v(t, x)$ as before.

4.2. Rescaled conservations of mass, entropy and moment. We revisit the conservation laws and the entropy functional for the rescaled equation (4.3).

Proposition 4.1. Let $\kappa=n(2-\alpha)>0$ and assume that the initial data $u_{0} \in L^{\alpha}\left(\mathbb{R}^{n}\right) \cap$ $L_{2}^{1}\left(\mathbb{R}^{n}\right)$ with $u_{0} \geq 0$. Let $(v, \phi)$ be a weak solution of (4.3) and set the functionals $W_{s}(v, \phi)$, $H(v(t))$ and $K_{s}(v, \phi)$ as follows:

$$
\begin{aligned}
& W_{s}(v, \phi)(t) \equiv \frac{1}{2} H(v(t))-\frac{1}{2} e^{-\kappa t} \int_{\mathbb{R}^{n}} v(t) \phi(t) d x \\
& H(v(t))=\frac{2}{\alpha-1} \int_{\mathbb{R}^{n}} v^{\alpha}(t) d x+\int_{\mathbb{R}^{n}}|x|^{2} v(t) d x, \\
& K_{s}(x, v(t), \phi(t)) \equiv \nabla\left(\frac{\alpha}{\alpha-1} v^{\alpha-1}+\frac{1}{2}|x|^{2}-e^{-\kappa t} \phi\right) .
\end{aligned}
$$

Then

$$
\begin{aligned}
& \int_{\mathbb{R}^{n}} v(t) d x=\int_{\mathbb{R}^{n}} u_{0}(x) d x, \\
& W_{s}(t)+\int_{0}^{t}\left[\int_{\mathbb{R}^{n}} v\left|K_{s}(x, v, \phi)(\tau)\right|^{2} d x d \tau+e^{-(\kappa+2) \tau} \int_{\mathbb{R}^{n}}|\nabla \phi(\tau)|^{2} d x\right] d \tau \leq W_{s}(0) .
\end{aligned}
$$

Proof. Let $\kappa=-(\sigma-n-2)=n(2-\alpha)>0$. Multiplying (1.9) by $\frac{\alpha}{\alpha-1} v^{\alpha-1}+\frac{1}{2}|x|^{2}-e^{-\kappa t} \phi$ and integrating by parts, we see that

$$
\begin{aligned}
\int_{\mathbb{R}^{n}} \partial_{t} v\left(\frac{\alpha}{\alpha-1} v^{\alpha-1}+\frac{1}{2}|x|^{2}\right. & \left.-e^{-\kappa t} \phi\right) d x \\
& =-\int_{\mathbb{R}^{n}} v\left|\nabla\left(\frac{\alpha}{\alpha-1} v^{\alpha-1}+\frac{1}{2}|x|^{2}-e^{-\kappa t} \phi\right)\right|^{2} d x
\end{aligned}
$$

The left hand side can be represented as

$$
\begin{aligned}
& \int_{\mathbb{R}^{n}} \partial_{t} v \cdot\left(\frac{\alpha}{\alpha-1} v^{\alpha-1}+\frac{1}{2}|x|^{2}-e^{-\kappa t} \phi\right) d x \\
& \quad=\frac{d}{d t}\left[\frac{1}{\alpha-1} \int_{\mathbb{R}^{n}} v^{\alpha} d x+\frac{1}{2} \int_{\mathbb{R}^{n}}|x|^{2} v d x-\int_{\mathbb{R}^{n}} e^{-\kappa t} v \phi d x\right]+\int_{\mathbb{R}^{n}} v \cdot \partial_{t}\left(e^{-\kappa t} \phi\right) d x
\end{aligned}
$$


For the elliptic part of the system

$$
\begin{aligned}
\int_{\mathbb{R}^{n}} v \cdot \partial_{t} \phi d x & =e^{-2 t} \int \nabla \phi \cdot \nabla \partial_{t} \phi d x+\lambda \int_{\mathbb{R}^{n}} \phi \cdot \partial_{t} \phi d x \\
& =\frac{1}{2} \frac{d}{d t} \int_{\mathbb{R}^{n}}\left(e^{-2 t}|\nabla \phi|^{2}+\lambda|\phi|^{2}\right) d x+e^{-2 t} \int_{\mathbb{R}^{n}}|\nabla \phi|^{2} d x
\end{aligned}
$$

and we obtain

$$
\begin{aligned}
\int_{\mathbb{R}^{n}} v \cdot \partial_{t}\left(e^{-\kappa t} \phi\right) d x= & \frac{1}{2} \frac{d}{d t} \int_{\mathbb{R}^{n}} e^{-\kappa t} v \phi d x+\kappa e^{-\kappa t} \int_{\mathbb{R}^{n}} v \phi d x \\
& +e^{-(\kappa+2) t} \int_{\mathbb{R}^{n}}|\nabla \phi|^{2} d x-\kappa e^{-\kappa t} \int_{\mathbb{R}^{n}} v \phi d x .
\end{aligned}
$$

Thus the left hand side of (4.6) is

$$
\begin{aligned}
& \frac{d}{d t}\left(\frac{1}{\alpha-1}\|v(t)\|_{\alpha}^{\alpha}+\frac{1}{2} \int_{\mathbb{R}^{n}}|x|^{2} v d x-\int_{\mathbb{R}^{n}} e^{-\kappa t} v \phi d x+\frac{1}{2} \int_{\mathbb{R}^{n}} e^{-\kappa t} v \phi d x\right) \\
& +e^{-(\kappa+2) t} \int_{\mathbb{R}^{n}}|\nabla \phi|^{2} d x \\
& =\frac{d}{d t} W_{s}(v, \phi)(t)+e^{-(\kappa+2) t} \int_{\mathbb{R}^{n}}|\nabla \phi|^{2} d x .
\end{aligned}
$$

Combining (4.6) and (4.7), we get

$$
\begin{gathered}
W_{s}(v, \phi)(t)+\int_{0}^{t}\left[\int_{\mathbb{R}^{n}} v(\tau)\left|K_{s}(x, v(\tau), \phi(\tau))\right|^{2} d x+e^{-(\kappa+2) \tau} \int_{\mathbb{R}^{n}}|\nabla \phi(\tau)|^{2} d x\right] d \tau \\
=W_{s}\left(u_{0}\right)
\end{gathered}
$$

Again the rigorous justification requires a regularization argument for the equation, and we obtain the inequality version of (4.8) as a consequence.

The following estimate is a direct consequence of the above a priori bound of the rescaled solution.

Proposition 4.2. Let $(v(t), \phi(t))$ be a weak solution of (4.3). in the case $1<\alpha \leq 2-\frac{2}{n}$ with small data: $\left\|u_{0}\right\|_{1} \leq C_{n}$.

(1) Then we have

$$
\|v(t)\|_{q} \leq C
$$

for all $1 \leq q \leq \infty$, and

(2) for all $n /(n-1)<r \leq \infty$,

$$
\|\nabla \phi(t)\|_{r} \leq C e^{2 t}
$$

Proof. The proof follows that of Lemma 2.2. Let $E_{\lambda, t}$ be the fundamental solution of $\left(-e^{-2 t} \Delta+\lambda\right)$ in $\mathbb{R}^{n}$. Then for $v \in L^{1}\left(\mathbb{R}^{n}\right) \cap L^{\alpha}\left(\mathbb{R}^{n}\right), \phi=E_{\lambda, t} * v$ is a solution of the second equation of the system (4.3). We have

$$
\frac{e^{-\kappa t}}{2}\left(e^{-2 t}\|\nabla \phi\|_{2}^{2}+\lambda\|\phi\|_{2}^{2}\right)=\int_{\mathbb{R}^{n}} e^{-\kappa t} v \phi d x \leq e^{-\kappa t}\left\|E_{\lambda, t}\right\|_{L_{w}^{\frac{n}{n-2}}}\|v\|_{1}^{1-\gamma}\|v(t)\|_{\alpha}^{1+\gamma}
$$

for any $\gamma=\frac{\alpha(n-2)}{n(\alpha-1)}-1<\alpha-1$. 
Indeed, by the Hölder inequality,

$$
\begin{aligned}
& \int_{\mathbb{R}^{n}} v \phi d x \leq\|v\|_{r}\|\phi\|_{r^{\prime}} \quad \text { for } \frac{1}{r}+\frac{1}{r^{\prime}}=1 \\
& \leq\|v\|_{1}^{1-\gamma}\|v\|_{\alpha}^{\gamma}\|\phi\|_{r^{\prime}} \quad\left(\frac{1}{r}=1-\gamma+\frac{\gamma}{\alpha}\right)
\end{aligned}
$$

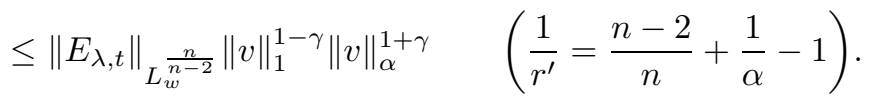

Under the assumption $\alpha \leq 2-\frac{2}{n}$,

$$
\alpha\left(1-\frac{1}{\alpha}\right) \leq(\gamma+1)\left(1-\frac{1}{\alpha}\right)=\frac{n-2}{n}
$$

and this gives $\gamma+1 \geq \alpha$. Hence noting that $\left\|E_{\lambda, t}\right\|_{n /(n-2)} \simeq C e^{2 t} \leq C e^{\kappa t}$, we have

$$
e^{-\kappa t} \int_{\mathbb{R}^{n}} v(t) \phi(t) d x \leq C\|v(t)\|_{1}^{1-\gamma}\|v(t)\|_{\alpha}^{1+\gamma}
$$

and

$$
\|v(t)\|_{\alpha}^{\alpha} \leq W_{s}(0)+C\left\|u_{0}\right\|_{1}^{1-\gamma}\|v(t)\|_{\alpha}^{1+\gamma}
$$

where $\gamma+1 \geq \alpha$. Therefore, under the smallness condition $\left\|u_{0}\right\|_{1}<C_{n}$, we obtain the bound

$$
\|v(t)\|_{\alpha}^{\alpha} \leq C
$$

uniform in $t$. Hence for $1 \leq q \leq 2-\frac{2}{n}$, the estimate (4.11), $L^{1}$ conservation law and the entropy bound

$$
W_{s}(t) \leq W_{s}(0)
$$

imply

$$
\frac{1}{2(\alpha-1)}\|v(t)\|_{\alpha}^{\alpha}+\frac{1}{2} \int_{\mathbb{R}^{n}}|x|^{2} v(t) d x \leq C\left(W_{s}(0)+C_{n}\left\|u_{0}\right\|_{1}^{\frac{\alpha(1-\gamma)}{\alpha-(\gamma+1)}}\right)
$$

for all $t \in[0, \infty)$, where $\gamma<\alpha-1$. Here we note that

$$
\frac{\alpha(1-\gamma)}{\alpha-(1+\gamma)}=\frac{n(\alpha-2)+2 \alpha}{n \alpha+2}
$$

For the case $q \geq 2-\frac{2}{n}$, the estimate is quite similar to that in the proof of Theorem 2.4. We apply the standard parabolic estimates to see that for any $q>\alpha$

$$
\begin{aligned}
\frac{d}{d t}\|v(t)\|_{q}^{q} & +n(q-1)\|v(t)\|_{q}^{q}+\frac{2 r}{\alpha+q-1}\left\|\nabla v^{\gamma}(t)\right\|_{2}^{2} \\
& =(q-1) e^{-\kappa t} \int_{\mathbb{R}^{n}} \nabla v^{r}(t) \cdot \nabla \phi(t) d x \leq C(q-1) e^{-(\kappa-2) t}\|v(t)\|_{q+1}^{q+1}(
\end{aligned}
$$

by the positivity of $(v, \phi)$, where $\gamma=\frac{1}{2}(\alpha+q-1)$. Noting $\kappa>2$ under $1<\alpha<2-\frac{2}{n}$, the very much similar argument in the proof of Theorem 2.4 implies

$$
\|v(t)\|_{q} \leq C\left(W_{s}(0),\left\|u_{0}\right\|_{1},\left\|u_{0}\right\|_{\infty}\right)
$$

for any $1 \leq q \leq \infty$, and we obtain the desired a priori estimate for $t \in[0, \infty)$. Note that one can eliminate the initial restriction on $\left\|u_{0}\right\|_{q}$ by the parabolic regularity argument. 
The estimate for the potential term $\phi$ directly follows from the estimate for $v(t)$ and the Hardy-Littlewood inequality: by $\nabla \phi=\left(-e^{-2 t} \Delta+1\right)^{-1} \nabla v$,

$$
\begin{aligned}
\|\nabla \phi\|_{q} & =\left\|\left(-e^{-2 t} \Delta+1\right)^{-1} \nabla v\right\|_{q} \\
& \leq C e^{2 t}\left\|\left(-e^{-2 t} \Delta+1\right)^{-1} e^{-2 t} \Delta v\right\|_{r} \\
& \leq C e^{2 t}\|v\|_{r} \leq C e^{2 t}
\end{aligned}
$$

with $q>n /(n-1)$ and

$$
\frac{1}{q}=\frac{1}{r}-\frac{1}{n}
$$

Once we obtain the above uniform bound for the rescaled solution, we can immediately obtain the time decay estimate for the solution of the original equation.

$$
\int_{\mathbb{R}^{n}} v^{q}\left(t^{\prime}, x^{\prime}\right) d x^{\prime}=\int_{\mathbb{R}^{n}} e^{n(q-1) t^{\prime}} u^{q}(t, x) d x=(1+\sigma t)^{(q-1) n / \sigma} \int_{\mathbb{R}^{n}} u^{q}(t, x) d x
$$

in the original variables $(t, x)$. Hence we obtain the following decay estimate for the original solution as a corollary of Proposition 4.2.

Proposition 4.3. Let $u_{0} \in L_{2}^{1}\left(\mathbb{R}^{n}\right) \cap L^{\infty}\left(\mathbb{R}^{n}\right)$ and $(u(t), \psi(t))$ be a weak solution of $(1.9)$. Then for $1<\alpha \leq 2-\frac{2}{n}$ with small initial data $\left\|u_{0}\right\|_{1}<\varepsilon$, we have

$$
\|u(t)\|_{q} \leq C(1+\sigma t)^{-\frac{n}{\sigma}\left(1-\frac{1}{q}\right)}
$$

for all $1 \leq q \leq \infty$

5. Asymptotic profile. The expected asymptotic profile of the decaying solution is governed by the principal term, and it corresponds to the Barenblatt solution of the single porous medium equation

$$
\partial_{t} u-\Delta u^{\alpha}=0, \quad x \in \mathbb{R}^{n}, t>0
$$

Definition. For $\alpha>1$, we let

$$
U(t, x) \equiv(1+\sigma t)^{-\frac{n}{\sigma}}\left[A-\frac{\alpha-1}{2 \alpha} \frac{|x|^{2}}{(1+\sigma t)^{2 / \sigma}}\right]_{+}^{1 /(\alpha-1)},
$$

where $\sigma=n(\alpha-1)+2$ and $A$ is chosen so that $\|U\|_{1}=M \equiv\left\|u_{0}\right\|_{1}$.

Applying the method of the transport equation or Fokker-Planck equation due to Carrillo-Toscani [8], we have the following result.

TheOREM 5.1. Let $\lambda>0$ and $1<\alpha<2-\frac{2}{n}$. Then for any positive initial data $u_{0} \in$ $L_{2}^{1}\left(\mathbb{R}^{n}\right) \cap L^{\infty}\left(\mathbb{R}^{n}\right)$, the decaying weak solution $u(t, x)$ in Proposition 4.3 with the small initial data satisfies the following asymptotic behavior:

$$
\|u(t)-U(t)\|_{1} \leq(1+\sigma t)^{-\nu}
$$

where $\sigma=n(\alpha-1)+2$ and $\nu=\min \left(\frac{2 \sigma}{(n+2) \alpha-n}, 1\right)$.

Here, we only give an outline of the proof of Theorem 5.1. The detailed version will appear elsewhere. We compute the time derivative of the second moment. For a weak 
solution $u$ and $\psi$ of (4.3) put

$$
\begin{aligned}
H(v(t)) & \equiv \int_{\mathbb{R}^{n}}|x|^{2} v(t) d x+\frac{2}{\alpha-1} \int_{\mathbb{R}^{n}} v^{\alpha}(t) d x \\
J(v(t)) & \equiv \int_{\mathbb{R}^{n}} v(t)\left|\nabla\left(\frac{\alpha}{\alpha-1} v^{\alpha-1}(t)+\frac{|x|^{2}}{2}\right)\right|^{2} d x \\
I(v(t)) & \equiv \int_{\mathbb{R}^{n}} v(t)\left|\nabla\left(\frac{\alpha}{\alpha-1} v^{\alpha-1}(t)+\frac{|x|^{2}}{2}-e^{-\kappa t} \phi(t)\right)\right|^{2} d x .
\end{aligned}
$$

It is now well understood that for a weak solution $v$ and $\phi$ of (4.3), the functional $H(v)$ plays the role of a metric in the solution spaces. Namely we have

$$
\begin{aligned}
|H(v(t))-H(v(s))| \leq & 2 \int_{s}^{t} J(v(\tau)) d \tau \\
& +\int_{s}^{t} e^{-\kappa \tau}\left[\int_{\mathbb{R}^{n}} e^{2 \tau} v^{\alpha+1}(\tau) d x+\int_{\mathbb{R}^{n}}|\phi(\tau)|^{2} d x\right] d \tau,
\end{aligned}
$$

where $\kappa=n(2-\alpha)$. In particular, for $1<\alpha<2-\frac{2}{n}$, we have that $H(v(t))$ is monotonically decreasing in $t$ and

$$
H(v(t)) \leq H\left(u_{0}\right), \quad t>0 .
$$

The inequality (5.5) follows in a similar way we derived (4.5) in Proposition 4.1. Under the condition $1<\alpha \leq 2-\frac{2}{n}$ we see $\kappa \geq 2$, and we have already seen that $\|v\|_{\alpha+1}^{\alpha+1} \leq C$ and $\|\phi\|_{2} \leq C$. Then it follows that

$$
H(v(t)) \leq C,
$$

and for some appropriate sequence $\left\{t_{n}\right\}$,

$$
\begin{aligned}
& \lim _{n \rightarrow \infty} H\left(v\left(t_{n}\right)\right)-H\left(u_{0}\right)+2 \int_{0}^{\infty} J(u(\tau)) d \tau \\
& \leq 2 \int_{0}^{\infty} e^{-\kappa \tau} \int_{\mathbb{R}^{n}}\left(|\phi(\tau)|^{2}+e^{2 \tau} v^{\alpha+1}(\tau)\right) d x d \tau<\infty, \\
& \left|H\left(v\left(t_{n}\right)\right)-H\left(v\left(t_{m}\right)\right)\right| \\
& \leq 2 \int_{t_{m}}^{t_{n}} J(u(\tau)) d \tau+2 \int_{t_{m}}^{t_{n}} e^{-\kappa \tau} \int_{\mathbb{R}^{n}}\left(|\phi(\tau)|^{2}+e^{2 \tau} v^{\alpha+1}(\tau)\right) d x d \tau \rightarrow 0, \quad n, m \rightarrow \infty,
\end{aligned}
$$

and this shows that $\left\{H\left(v\left(t_{n}\right)\right)\right\}$ is a Cauchy sequence for $n \rightarrow \infty$.

On the other hand, one may observe that the functional $I(v)$ decays exponentially in time. Since $2(\kappa-2)=2 n(2-\alpha)-4>0$ under the condition $\alpha<2-\frac{2}{n}$, we choose $\eta$ such that $\nu \equiv 2-\eta<\min (2(\kappa-2), 1)$ and it follows that

$$
I(v(t)) \leq e^{-\nu t}\left(I\left(v_{0}\right)+C \int_{0}^{\infty} e^{(\nu-2(\kappa-2)) \tau} d \tau\right) .
$$

This is obtained by the direct estimate for the functional $I(v)$ with the aid of the regularity of solutions. The critical type of the Sobolev inequality (cf. [27]) is required to estimate the nonlinear interaction.

On the other hand, choosing a suitable subsequence $t_{n},\left\{H\left(v\left(t_{n}\right)\right)\right\}$ is a Cauchy sequence and there exists a constant $H_{\infty}$ such that $\lim _{n \rightarrow \infty} H\left(v\left(t_{n}\right)\right)=H_{\infty}$. Moreover, 
we conclude that there exists a limit function $V$ in $X=W^{1, \alpha} \cap L_{2}^{1}\left(\mathbb{R}^{n}\right)$ such that $v\left(t_{n}\right) \rightarrow V, t_{n} \rightarrow \infty$ in $X$. By (5.7) $I\left(v\left(t_{n}\right)\right) \rightarrow 0(n \rightarrow \infty)$, we have $I(V)=0$ and we obtain $\nabla V^{\alpha-1}=-\frac{\alpha-1}{\alpha} x$. This follows by recalling $M=\left\|u_{0}\right\|_{1}$,

$$
V(x)=\left[A-\frac{\alpha-1}{2 \alpha}|x|^{2}\right]_{+}^{1 /(\alpha-1)},
$$

where $A$ is chosen such that the $L^{1}$ norm of $V(x)$ is normalized as $M$. Again by estimate (5.5) we have

$$
\begin{aligned}
0 & \leq H(v(t))-H\left(v_{\infty}\right) \\
& \leq-2 \int_{t}^{\infty} I(v(\tau)) d \tau+\int_{t}^{\infty} e^{-\kappa \tau}\left[\int_{\mathbb{R}^{n}} e^{2 \tau} v^{\alpha+1}(\tau) d x+\int_{\mathbb{R}^{n}}|\phi(\tau)|^{2} d x\right] d \tau,
\end{aligned}
$$

and using the very much similar argument in [8], the desired estimate

$$
\|v(t)-V\|_{1} \leq C e^{-\nu t}
$$

follows. This gives the result of Theorem 5.1 by change of the variable into the original variables.

The entire proof relies on the regularity theorem of degenerate parabolic equations and the crucial estimate for $I(v)$ requires some estimates. The detailed discussion will be published elsewhere.

Acknowledgments. The author is grateful for a fruitful discussion with Professors Masashi Misawa, Toshitaka Nagai, Masaki Kurokiba and Yoshie Sugiyama. He also thanks the referee for pointing out some unclear parts. This work is partially supported by JSPS grant in aid scientific research B \#15340056.

\section{References}

[1] P. Biler, Existence and nonexistence of solutions for a model of gravitational interaction of particles, III, Colloq. Math. 68 (1995), 229-239.

[2] P. Biler, Local and global solvability of some parabolic systems modeling chemotaxis, Adv. Math. Sci. Appl. 8 (1998), 715-743.

[3] P. Biler and J. Dolbeault, Long time behavior of solutions to Nernst-Planck and DebyeHückel drift-diffusion systems, Ann. Henri Poincaré 1 (2000), 461-472.

[4] P. Biler, W. Hebisch and T. Nadzieja, The Debye system: existence and large time behavior of solutions, Nonlinear Anal. T.M.A. 23 (1994), 1189-1209.

[5] P. Biler, G. Karch, Ph. Laurençot and T. Nadzieja, The $8 \pi$-problem for radially symmetric solutions of a chemotaxis model in a disc, Topol. Methods Nonlinear Analysis, to appear.

[6] P. Biler, T. Nadzieja and R. Stańczy, Nonisothermal systems of self-attracting Fermi-Dirac particles, Banach Center Publ. 66 (2004), 61-78.

[7] L. A. Caffarelli and L. C. Evans, Continuity of the temperature in two-phase Stefan problem, Arch. Rat. Mech. Anal. 81 (1983), 199-220.

[8] J. A. Carrillo and G. Toscani, Asymptotic $L^{1}$ decay of solutions of the porous medium equation to self-similarity, Indiana Univ. Math. J. 49 (2000), 113-142. 
[9] J. Dolbeault and B. Perthame, Optimal critical mass in the two-dimensional Keller-Segel model in $\mathbb{R}^{2}$, C. R. Math. Acad. Sci. Paris 339 (2004), 611-616.

[10] H. Fujita, On blowing up of solutions of the Cauchy problem for $u_{t}=\Delta u+u^{1+\alpha}$, J. Fac. Sci. Univ. Tokyo Sect. I 13 (1966), 35-37.

[11] Y. Giga and R. Kohn, Asymptotically self-similar blow-up of semilinear heat equations, Comm. Pure Appl. Math. 38 (1985), 297-319.

[12] W. Jäger and S. Luckhaus, On explosions of solutions to a system of partial differential equations modeling chemotaxis, Trans. Amer. Math. Soc. 329 (1992), 819-824.

[13] A. Jüngel, Qualitative behavior of solutions of a degenerate nonlinear drift-diffusion model for semiconductors, Math. Model. Meth. Appl. Sci. 5 (1995), 497-518.

[14] E. F. Keller and L. A. Segel, Initiation of slime mold aggregation viewed as an instability, J. Theor. Biol. 26 (1970), 399-415.

[15] H. Kozono, T. Ogawa and Y. Taniuchi, The critical Sobolev inequalities in Besov spaces and regularity criterion to some semi-linear evolution equations, Math. Z. 242 (2002), 251-278.

[16] M. Kurokiba, T. Nagai and T. Ogawa, The uniform boundedness and threshold for the global existence of the radial solution to a drift-diffusion system, Comm. Pure Appl. Anal. 5 (2006), 97-106.

[17] M. Kurokiba and T. Ogawa, Finite time blow-up of the solution for a nonlinear parabolic equation of drift-diffusion type, Diff. Integral Equations 16 (2003), 427-452.

[18] S. Luckhaus and Y. Sugiyama, Asymptotic profile with the optimal convergence rate for a parabolic equation of chemotaxis in super-critical cases, Indiana Univ. J. Math., to appear.

[19] M. S. Mock, An initial value problem from semiconductor device theory SIAM J. Math. Anal. 5 (1974), 597-612.

[20] T. Nagai, Blow-up of radially symmetric solutions to a chemotaxis system, Adv. Math. Sci. Appl. 5 (1995), 581-601.

[21] T. Nagai, Global existence of solutions to a parabolic system for chemotaxis in two space dimensions, Nonlinear Anal. T.M.A. 30 (1997), 5381-5388.

[22] T. Nagai, Blowup of nonradial solutions to parabolic-elliptic systems modeling chemotaxis in two-dimensional domains, J. Inequal. Appl. 6 (2001), 37-55.

[23] T. Nagai and T. Ogawa, Brezis-Merle inequalities and application to the global existence of the Cauchy problem of the Keller-Segel and self-interacting systems, preprint.

[24] T. Nagai, T. Senba and T. Suzuki, Chemotactic collapse in a parabolic system of mathematical biology, Hiroshima Math. J. 30 (2000), 463-497.

[25] T. Nagai, T. Senba and K. Yoshida, Application of the Trudinger-Moser inequality to a parabolic system of chemotaxis, Funkcial. Ekvac. 40 (1997), 411-433.

[26] T. Nagai, R. Syukuinn and M. Umesako, Decay property and asymptotic profile to a parabolic system of chemotaxis in $\mathbb{R}^{n}$, Funkcial. Ekvac. 46 (2003), 383-407.

[27] T. Ogawa and Y. Taniuchi, Critical Sobolev inequality and uniqueness problem to the Navier-Stokes equations, Tohoku Math. J. 56 (2004), 65-77.

[28] T. Ogawa and Y. Tsutsumi, Blow-up of $H^{1}$ solutions for the one-dimensional nonlinear Schrödinger equation with critical power nonlinearity, Proc. Amer. Math. Soc. 111 (1991), 487-496.

[29] F. Otto, The geometry of dissipative evolution equations: the porous medium equation, Comm. P.D.E. 26 (2001), 101-174. 
[30] T. Senba and T. Suzuki, Chemotactic collapse in a parabolic-elliptic system of mathematical biology, Adv. Differential Equations 6 (2001), 21-50.

[31] T. Senba and T. Suzuki, Weak solutions to a parabolic elliptic system of chemotaxis, J. Funct. Anal. 191 (2002), 17-51.

[32] E. Stein, Singular Integrals and Differentiability Properties of Functions, Princeton Univ. Press, Princeton, New Jersey, 1970.

[33] Y. Sugiyama, Global existence in sub-ctitical case and finite time blow up in super-critical cases to degenerate Keller-Segel systems, Diff. Integral Equations, to appear.

[34] Y. Sugiyama, Application of the best possible constant of the Sobolev inequality to degenerate Keller-Segel models, Adv. Diff. Equations, to appear.

[35] Y. Sugiyama and H. Kunii, Global existence and decay properties for a degenerate KellerSegel model with a power foctor in drift term, J. Differential Equations 227 (2006), 333364 .

[36] T. Suzuki, Method of blow up envelope and applications, in: Asymptotic Analysis and Singularities, Adv. Studies Pure Math. (2006), to appear.

[37] G. Wolansky, On steady distributions of self-attracting clusters inuder friction and fluctuations, Arch. Rat. Mech. Anal. 119 (1992), 355-391.

[38] G. Wolansky, On the evolution of self-interacting clusters and applications to semilinear equations with exponential nonlinearity, J. Anal. Math. 59 (1992), 251-272.

[39] F. Weissler, Existence and non-existence of global solutions for a semilinear heat equation, Israel J. Math. 38 (1981), 29-40.

[40] A. Yagi, Norm behavior of solutions to a parabolic system of chemotaxis, Math. Japon. 45 (1997), 241-265. 